\title{
RELAÇÃO ENTRE A POSTURA ESTÁTICA DA COLUNA VERTEBRAL DE CRIANÇAS E SUA FLEXIBILIDADE
}

\author{
Juliana Adami Sedrez ${ }^{1}$ \\ Marja Bochehin do Valle ${ }^{2}$ \\ Eduardo Bojunga Corrêa de Oliveira ${ }^{3}$ \\ Cláudia Tarragô Candotti ${ }^{4}$
}

SEDREZ, J. A.; VALLE M. B. do; OLIVEIRA, E. B. C. de; CANDOTTI, C. T. Relação entre a postura estática da coluna vertebral de crianças e sua flexibilidade. Arq. Cienc. Saúde UNIPAR, Umuarama, v. 22, n. 2, p, 69-74, maio/ago. 2018.

\begin{abstract}
RESUMO: A coluna vertebral é um segmento complexo e a sua flexibilidade é importante para o aumento da resistência às forças de pressão e para a manutenção da postura corporal. No entanto, ainda não está clara, na literatura, a relação entre a postura estática da coluna vertebral com a sua flexibilidade. Verificar se a postura estática da coluna vertebral está relacionada com a sua flexibilidade, bem como o comportamento dessas variáveis entre os sexos. Participaram deste estudo 36 sujeitos de 7 a 18 anos de idade, que realizaram raios-x panorâmicos da coluna vertebral na incidência perfil direito. Nas radiografias foram medidos os ângulos de Cobb, das curvaturas torácica e lombar. A flexibilidade da coluna vertebral foi verificada por meio do Flexicurva. A avaliação consistiu na demarcação dos pontos anatômicos T1, T6 e T12 para a cifose torácica e T12, L4 e S2 para lordose lombar. Após, o Flexicurva foi moldado no dorso dos indivíduos durante três momentos: (a) com coluna neutra, (b) com coluna flexionada e (c) com coluna estendida, tanto para a coluna lombar quanto torácica. Em seguida, o contorno do dorso foi transcrito para um papel milimetrado. A partir das medidas obtidas, foi possível obter a flexibilidade total da coluna, bem como de flexão e extensão, além dos índices de cifose torácica e lordose lombar. Foram utilizados os testes de Correlação Produto-Momento de Pearson, o Coeficiente Correlação de Spearman e MANOVA $(\alpha<0,05)$. Não foram encontradas correlações entre as ADMs da coluna vertebral com os ângulos de Cobb de cifose e lordose. No entanto, foram encontradas correlações variando de moderada a alta entre as ADMs torácicas e lombares com os índices dessas regiões. A comparação entre os sexos demonstrou que os meninos possuem maiores valores de ADM de flexão lombar enquanto as meninas possuem maiores valores de ADM de flexão torácica. Não é possível afirmar que o ângulo de Cobb de cifose e lordose está relacionado com a flexibilidade da coluna vertebral, no entanto, os índices dessas regiões parecem ser um bom indicativo da flexibilidade.
\end{abstract}

PALAVRAS-CHAVE: Amplitude de Movimento Articular. Maleabilidade. Postura.

\section{RELATIONSHIP BETWEEN THE STATIC POSTURE OF CHILDREN'S SPINE AND FLEXIBILITY}

ABSTRACT: The spine is a complex segment and its flexibility is important to increase the resistance to pressure forces and for the maintenance of the body posture. However, the literature is still unclear on the relationship between the spine's static posture and its flexibility. Verify if the spine's static posture is related to its flexibility, as well as the behavior of those variables between genders. A total of 36 subjects aged 7 to 18 took part in this study. Panoramic x-rays of the spine in the right sagittal plan were obtained from the subjects. The thoracic and lumbar curvatures were measured using Cobb's angle in the radiographs and the spine's flexibility was verified by using Flexicurva. The assessment consisted in marking anatomical points T1, T6 and T12 for thoracic kyphosis and T12, L4 and S2 for lumbar lordosis. Then, the Flexicurva device was molded on the back of the subjects at three moments: (a) with a neutral spine, (b) with a flexed spine, and (c) with an extended spine, both for the lumbar and the thoracic spine. The contour of the back was then transcribed on to a graph paper. From the obtained measurements, it was possible to calculate the total flexibility of the spine, as well as during its flexion and extension, in addition to the thoracic kyphosis and lumbar lordosis indexes. In order to verify the correlation between the data, the Pearson product-moment correlation coefficient, Spearman correlation coefficient, and MANOVA $(\alpha<0.05)$ tests were used. No correlations were found between spinal ROMs with kyphosis and lordosis Cobb angles. However, correlations ranging from moderate to high between the thoracic and lumbar ROMs were found with the index obtained from those regions. The comparison between genders showed that male subjects present higher values of lumbar flexion ROM while female subjects have higher thoracic flexion ROM values. It is not possible to conclude that the kyphosis and lordosis Cobb's angle is related to the flexibility of the spine; however, the indexes obtained from those regions seem to be a good indication of the spine flexibility.

KEYWORDS: Articular. Pliability. Posture. Range of Motion.

\section{Introdução}

A coluna vertebral é um segmento de notável relevância funcional, já que fornece suporte e mobilidade ao corpo, além de fornecer proteção ao sistema nervoso central (BYRD; COMISKEY, 2007), sendo que a manutenção da sua posição fisiológica desempenha um papel fundamental sobre o controle do centro de gravidade e o equilíbrio corpo- ral (KADONO, et al., 2017), além de ser considerado importante para a preservação da flexibilidade da própria coluna vertebral e dos segmentos conexos (MALMSTRÖM, et al., 2015).

A relação inversa também tem sido proposta, onde tem sido sugerido que níveis adequados de flexibilidade são um dos fatores responsáveis pela manutenção de posições corporais adequadas (MALMSTRÖM, et al., 2015; COE-

DOI: 10.25110 /arqsaude.v22i2.2018.6224

${ }^{1}$ Graduada em Fisioterapia pela Universidade Católica de Pelotas e doutoranda em Ciência do Movimento Humano pela Universidade Federal do Rio Grande do Sul (UFRGS)

${ }^{2}$ Graduada em Educação Física pela Pontifícia Universidade Católica do Rio Grande do Sul e doutoranda em Ciência do Movimento Humano pela UFRGS ${ }^{3}$ Graduado em Educação Física pela UFRGS

${ }^{4}$ Doutora em Ciência do Movimento Humano pela UFRGS e docente do curso de fisioterapia e educação física, mestrado e doutorado da UFRGS. Graduado em Educação Física pela UFRGS

Autor correspondente: Juliana Adami Sedrez. Endereço: Av. Domingos de Almeida, 2187. Bairro Areal - Pelotas/RS. CEP: 96085-470. E-mail: julianasedrez@gmail.com 
LHO et al., 2014; LEMOS; SANTOS; GAYA, 2012), uma vez que a flexibilidade é uma das características do sistema muscular que promove eficiência de movimento, melhor desempenho muscular e prevenção de algumas patologias musculoesqueléticas (PENHA; JOÃO, 2008). Dessa maneira, percebe-se que essas variáveis parecem se inter-relacionar, gerando influências recíprocas.

Ainda que já se tenha um conhecimento acerca dessa relação entre a flexibilidade e a prevenção de alterações posturais, pouco se conhece como se dá essa relação durante a infância e adolescência (WIDHE, et al., 2001). Sabendo-se que nas fases da infância e da adolescência ocorrem diversas modificações em virtude da aceleração do crescimento e que esse estágio pode estar associado a variações de postura, torna-se fundamental a prevenção dos problemas posturais nesses períodos, sendo necessário, para isso, conhecer os fatores predisponentes a essas alterações (LEMOS; SANTOS; GAYA, 2012). Portanto, nesse contexto, torna-se relevante investigar essa relação nessa população. Dessa maneira, o objetivo desse estudo foi verificar se a postura estática da coluna vertebral está relacionada com a sua flexibilidade, bem como, verificar o comportamento dessas variáveis entre os sexos.

\section{Métodos}

Trata-se de um estudo transversal, cujo tamanho amostral foi definido utilizando o software Gpower 3.1.7, com base na família de testes z (correlação de Pearson) admitindo um teste bicaudal, assumindo como hipótese nula uma correlação muito fraca $(\mathrm{r}=0,1)$, uma expectativa de correlação moderada $(\mathrm{r}=0,5)$, um $\alpha=0,05$ e um poder de $80 \%$, resultando em uma amostra mínima de 34 participantes.

Foram avaliadas 36 crianças, com média de idade $12,2 \pm 2,6$ anos, sendo $50 \%(\mathrm{n}=18)$ do sexo masculino. Houve perda de duas crianças na análise da região lombar e uma na análise da região torácica, devido a problemas na coleta com o instrumento Flexicurva.

Foram incluídas crianças com idade entre 7 e 18 anos que possuíam condições de permanecer em ortostase sem auxílio e apresentavam requisição médica para exame de radiografia da coluna vertebral. Os critérios de exclusão foram ter histórico de intervenção cirúrgica na coluna vertebral ou apresentar disfunções osteomusculares importantes.

Este estudo foi aprovado pelo comitê de ética da Universidade onde foi realizado $\left(\mathrm{n}^{\circ}\right.$ 19685) e respeitou o recomendado pela Declaração de Helsink de 1975, revisada em 2000 e a Resolução 466/2012 do Conselho Nacional de Saúde do Brasil. As crianças só foram incluídas se concordavam com sua participação e se o representante legal assinasse o termo de consentimento livre e esclarecido.

\section{Procedimento de coleta e análise}

Cada criança participou de três avaliações realizadas no mesmo dia, seguindo sempre a mesma ordem: (a) radiografia digital panorâmica da coluna vertebral na incidência perfil direito, que possibilitou a obtenção dos ângulos de Cobb das curvaturas torácica e lombar; (b) avaliação da flexibilidade da coluna vertebral com o instrumento Flexicurva; e (c) avaliação morfológica das curvaturas da coluna vertebral utilizando o Flexicurva.
As radiografias foram realizadas em um hospital por um técnico em radiologia. A criança permanecia em ortostase, com flexão dos ombros e cotovelos, a fim de evitar a sobreposição do úmero na coluna vertebral.

A partir das radiografias digitais, avaliadores experientes realizaram cálculos dos ângulos de Cobb no software MATLAB ${ }^{\circledR}$ 7.9. Para o cálculo da cifose torácica foram marcados o platô vertebral superior de T1 e o platô vertebral inferior de T12 (CIL, et al., 2005) e, para lordose lombar, o platô vertebral superior de L1 e o platô vertebral inferior de L5 (GONÇALVES; PEREIRA, 2008). No entanto, se as extremidades das vértebras selecionadas não estivessem bem nítidas, as vértebras adjacentes imediatamente acima ou abaixo foram usadas como alternativas para definir o ângulo da curvatura. Todos os cálculos do ângulo Cobb foram realizados por dois avaliadores independentes e quando as medidas entre os avaliadores diferiram mais do que $5^{\circ}$, um terceiro avaliador realizava uma nova avaliação. Para as análises estatísticas foram utilizados os valores médios dos avaliadores.

Para as avaliações da flexibilidade da coluna vertebral, com o Flexicurva, utilizou-se o protocolo de Burton (1986), estando as crianças com as costas despidas, sendo palpados e marcados com adesivos os processos espinhosos (PE) de T1, T6 e T12 para a cifose torácica e T12, L4 e S2 para lordose lombar. As crianças eram avaliadas em três posições: (a) com coluna neutra, (b) com coluna flexionada e (c) com coluna estendida, tanto para a coluna lombar quanto torácica.

Para a avaliação com o Flexicurva na posição neutra, as crianças eram posicionadas de forma semelhante à avaliação radiográfica, em ortostase, em sua postura habitual, ombros e cotovelos fletidos, seguindo o protocolo de descrito por Oliveira et al. (2012) (Figura 1a).

Para avaliação da coluna lombar em flexão a criança encontrava-se sentada, com os pés apoiados no solo e realizava a flexão máxima da lombar (Figura 1b). Para a avaliação da coluna lombar em extensão a criança era posicionada em decúbito ventral, com os membros superiores apoiados no solo e realizava a extensão máxima da lombar, mantendo apoio das espinhas-ilíacas anterossuperiores (Figura 1c).

Para avaliação da flexibilidade da coluna torácica em flexão a criança permaneceu sentada, com os pés apoiados no solo e realizava a flexão máxima da torácica (Figura 1d). Para a avaliação da flexibilidade da coluna torácica em extensão a criança foi posicionada em ortostase, com os membros superiores apoiados em uma parede e realizava a extensão máxima da coluna torácica sem movimentar o quadril (Figura 1e). 
Figura 1: Posturas utilizadas para avaliação da flexibilidade da coluna vertebral em: (a) posição neutra, (b) flexão lombar, (c) extensão lombar, (d) flexão torácica, e (e) extensão torácica.

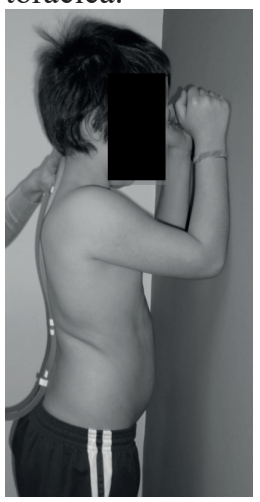

(a)

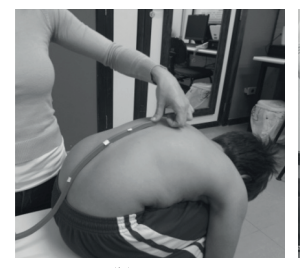

(b)

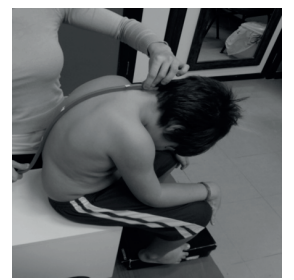

(d)

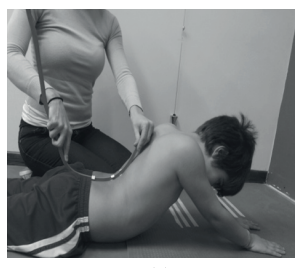

(c)

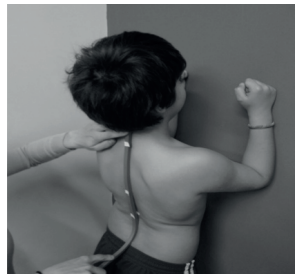

(e)
Em cada uma dessas posições o Flexicurva foi moldado na coluna vertebral e, na sequência foi cuidadosamente retirado do contato com a pele e posicionado sobre um papel milimetrado onde eram desenhadas as curvaturas e os PE demarcados. Para cada análise, 10 pares de coordenadas (x, y) foram marcados na curva, sendo elas: os três PE demarcados (T1, T6 e T12, para avaliação torácica; e T12, L4 e S2, para avaliação lombar), além de outros sete pontos aleatórios que representam a curvatura avaliada (um superior ao primeiro $\mathrm{PE}$ de referência, três pontos entre o primeiro e o segundo $\mathrm{PE}$ de referência, dois pontos entre o segundo e o terceiro PE de referência e um ponto abaixo do último $\mathrm{PE}$ de referência) (Figura 2).

Figura 2: Transcrição do contorno do Flexicurva para o papel milimetrado (a) na posição neutra, (b) em flexão lombar e (c) em extensão lombar.

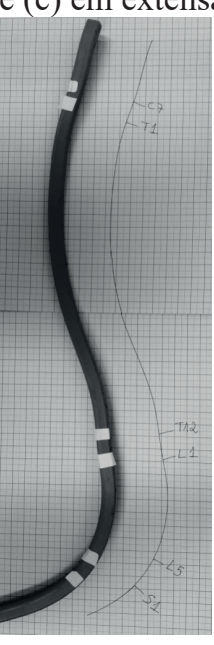

(a)

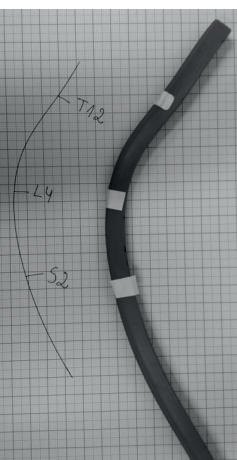

(b)

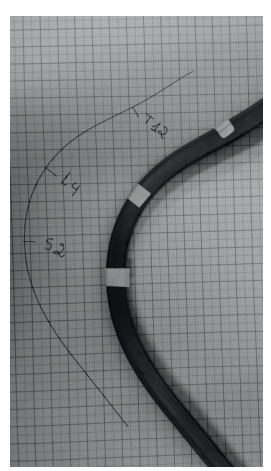

(c)
Os valores das coordenadas $(\mathrm{x}, \mathrm{y})$ de cada um desses pontos foram usados como dados de entrada para um algoritmo desenvolvido no software MATLAB ${ }^{\circledR} 7.9$ para a obtenção dos ângulos das curvaturas da coluna torácica e lombar em cada movimento. O algoritmo produz os ângulos de curvatura entre os pontos que representam o PE de T1 a T12 para a curvatura torácica e de T12 a S2 para a curvatura lombar.

Para a obtenção do ângulo de flexão, foi subtraído o valor angular na posição flexão máxima com valor angular na posição neutra. Os valores de extensão foram calculados subtraindo o valor angular na posição extensão máxima com valor angular na posição neutra, enquanto para os valores na amplitude total foram somados os valores de flexão e extensão (BURTON, 1986).

Para a avaliação morfológica das curvaturas da coluna vertebral com o Flexicurva, foi utilizado o contorno da coluna obtido durante a avaliação na posição neutra (Figura 1a). A avaliação das curvaturas foi realizada a partir dos índices de cifose e lordose (HINMAN, 2004). Para o cálculo dos índices foi traçada uma reta unindo $\mathrm{C} 7$ a $\mathrm{S} 1$, sendo que o ponto onde esta linha interseccionou a curva do contorno da coluna vertebral, marcou a transição entre as curvas torácica e lombar. A partir disso, foram obtidos os valores de largura e comprimento das curvas torácica e lombar (em centímetros), os quais são utilizados para calcular os índices de cifose e lordose, conforme descrito por Hinman (2004) (Figura 3).

Figura 3: (a) Obtenção dos valores de largura e comprimento torácico e lombar e (b) cálculo dos índices de cifose e lordose. (a)

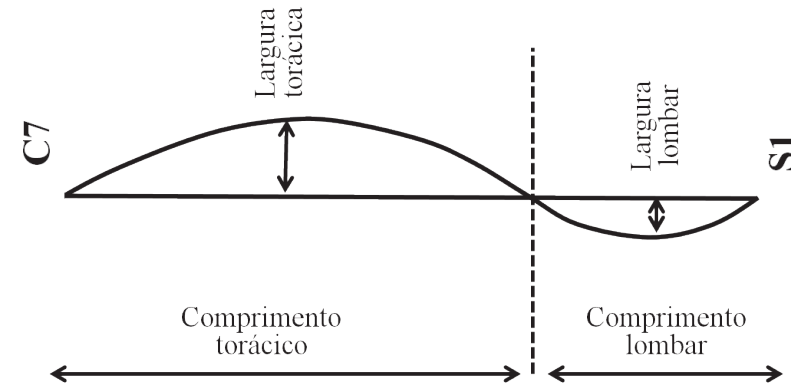

(b)

$$
\text { Índice }=\frac{\text { largura }}{\text { comprimento }} \times 100
$$

\section{Análise estatística}

A análise estatística foi realizada no software SPSS 20.0, sendo utilizado o teste Shapiro-Wilk para verificar a normalidade dos dados e uma análise descritiva com medidas de tendência central e dispersão. Além disso, os dados paramétricos foram submetidos ao Coeficiente de Correlação Produto-Momento de Pearson e os dados não paramétricos foram analisados com o Coeficiente de Correlação de Spearman. Uma análise de multivariância (MANOVA) foi utilizada para verificar possíveis diferenças entre os sexos. Em todas as análises foi adotado um $\alpha<0,05$.

As correlações (valores de $\mathrm{r}$ e rho) foram classificadas como muito fraca $(<0,1)$, fraca $(0,1-0,3)$, moderada $(0,3-0,5)$, alta $(0,5-0,7)$, muito alta $(0,7-0,9)$ e praticamente perfeita (entre 0,9 e 1), de acordo com Hopkins em Kotrlik e Williams (2003).

\section{Resultados}

Os dados descritivos da região torácica demonstram ângulos Cobb de 49,7 $\pm 11,6^{\circ}$, índice de cifose de 10,3 $\pm 2,4 \%$, ADM de flexão de $16,3 \pm 8,2^{\circ}, \mathrm{ADM}$ de extensão de $7,7 \pm 1,8^{\circ}$ e ADM total de $28,4 \pm 11,9^{\circ}$. Não foram encontradas corre- 
lações entre as ADMs da coluna torácica com o ângulo de Cobb de cifose torácica. No entanto, foi encontrada correlação moderada, negativa e significativa entre o índice de cifose e a ADM de flexão da coluna torácica e correlação moderada, positiva e significativa entre o índice de cifose e a ADM de extensão torácica.

Para a região lombar foi verificado ângulo de Cobb de $43,1 \pm 11,2^{\circ}$, índice de lordose de $9,3 \pm 3,4 \%$, ADM de flexão de $60,4 \pm 14,8^{\circ}$, obtendo-se correlação alta, positiva e sig- nificativa com o índice de lordose, indicando que conforme há incremento na flexibilidade lombar em flexão há um aumento do índice de lordose. A ADM de extensão lombar foi de $8,8 \pm 1,7^{\circ}$, apresentando correlação moderada, negativa e significativa com o índice de lordose. E, a ADM total foi de $72,2 \pm 12,9^{\circ}$, com correlação moderada, positiva e significativa com o índice de lordose. Não foram encontradas correlações entre as ADMs da coluna lombar com o ângulo de Cobb de lordose lombar.

Tabela 1: Correlação entre postura estática e flexibilidade da coluna vertebral.

\begin{tabular}{lcccccc}
\hline ADM Cifose & \multicolumn{3}{c}{ Ângulo de Cifose $(\mathrm{n}=36)$} & \multicolumn{3}{c}{ Índice de Cifose $(\mathrm{n}=35)$} \\
\cline { 2 - 6 } & $\mathrm{r}$ & rho & $\mathrm{p}$ & $\mathrm{r}$ & $\mathrm{rho}$ & $\mathrm{p}$ \\
Flexão & $-0,126$ & - & 0,465 & $-0,484$ & - & $0,003 *$ \\
Extensão & - & $-0,009$ & 0,958 & - & 0,417 & $0,013 *$ \\
Total & $-0,071$ & - & 0,682 & $-0,013$ & - & 0,939
\end{tabular}

\begin{tabular}{lcccccc} 
ADM Lombar & \multicolumn{3}{c}{ Ângulo de lordose $(\mathrm{n}=34)$} & \multicolumn{3}{c}{ Índice de Lordose $(\mathrm{n}=33)$} \\
\cline { 2 - 7 } & $\mathrm{r}$ & rho & $\mathrm{p}$ & $\mathrm{r}$ & rho & 0 \\
Flexão & 0,262 & - & 0,134 & 0,650 & - & $<0,001^{*}$ \\
Extensão & - & $-0,084$ & 0,638 & - & $-0,435$ & $0,011^{*}$ \\
Total & 0,178 & - & 0,313 & 0,397 & - & $0,022^{*}$ \\
\hline
\end{tabular}

*Correlação significativa

A flexibilidade da coluna vertebral, quando analisada separadamente pelo sexo, é diferente entre meninos e meninas para as ADMs de flexão, tanto na região torácica quanto lombar, bem como na ADM total da região torácica
(Tabela 2). As meninas apresentaram maior flexibilidade na região torácica e os meninos na região lombar. Quanto as variáveis da postura estática da coluna vertebral, não houve diferença entre os sexos.

Tabela 2: Comparação entre os sexos das variáveis da postura estática e da flexibilidade da coluna vertebral.

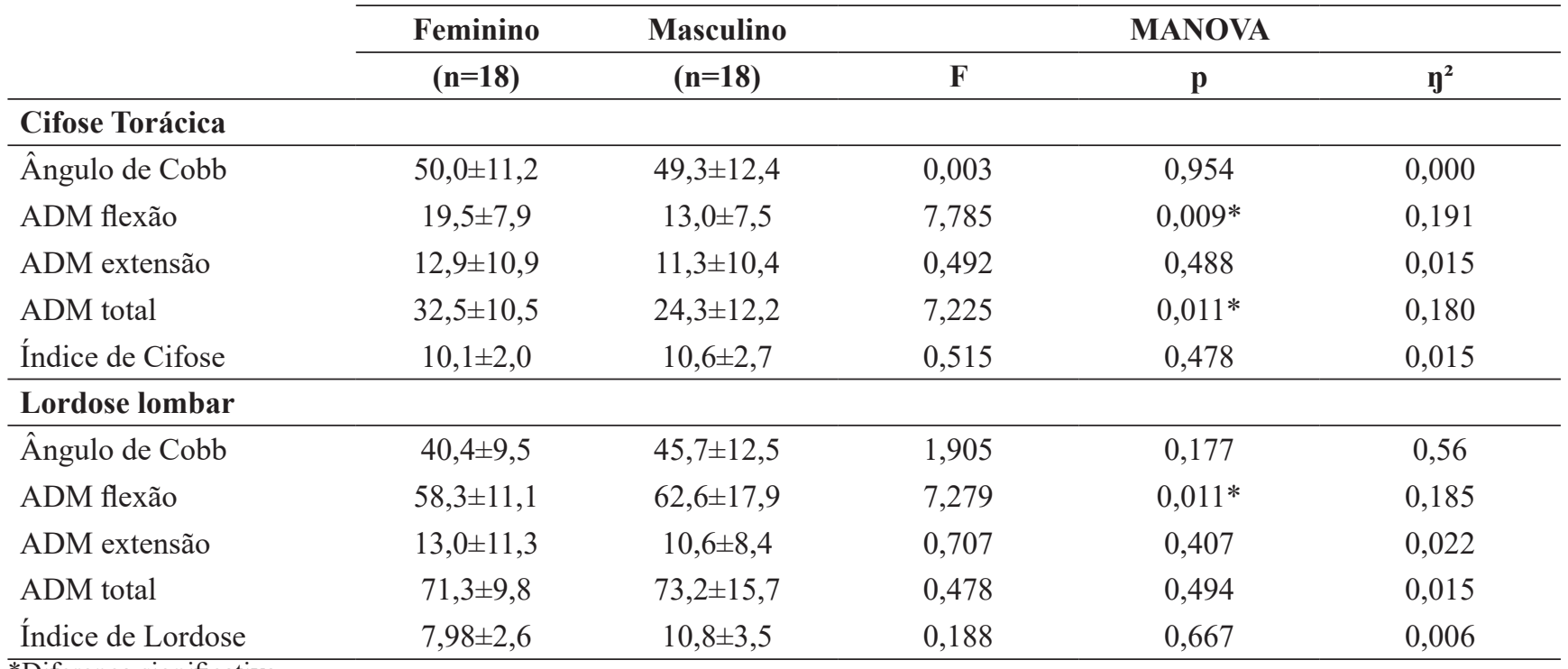

*Diferença significativa

\section{Discussão}

Em média as curvaturas sagitais da coluna vertebral dos jovens apresentaram valores angulares dentro dos padrões de normalidade estabelecidos tanto para cifose torácica, quanto para lordose lombar, sendo os valores angulares normais compreendidos no intervalo de $20^{\circ}$ a $50^{\circ} \mathrm{Cobb}$ para cifose torácica (BOSEKER et al., 2000), e de $31^{\circ}$ a $49,5^{\circ}$ Cobb para lordose lombar (PROPST-PROCTOR; BLECK; 1983). Não é possível estabelecer a normalidade dos indi- víduos avaliados quanto à flexibilidade da coluna vertebral, visto que ainda não se conhecem os valores de referência para essa avaliação quando se utiliza o instrumento Flexicurva. Essa carência limita as discussões sobre esse aspecto e pode ser considerada uma perspectiva para investigações futuras.

Não foram encontradas correlações entre as ADMs da coluna vertebral com os ângulos de Cobb de cifose e lordose. No entanto, foram encontradas correlações variando de moderada à excelente entre as ADMs da cifose torácica 
e lordose lombar com os seus respectivos índices. Essas correlações foram negativas entre o índice de cifose e a flexão torácica e entre o índice de lordose e a extensão lombar e positivas entre o índice de cifose e a extensão torácica e entre o índice de lordose e a flexão lombar. Esses resultados indicam que ao avaliar a coluna torácica com os índices (que corresponde a uma medida externa), quanto maior a curvatura estática mais o indivíduo consegue gerar extensão torácica e menos consegue flexionar essa região. Mas o oposto ocorre na lombar, em que o indivíduo com maior curvatura lombar apresenta maior capacidade de gerar flexão lombar e menor extensão lombar.

Lemos, Santos e Gaya (2012) que também avaliaram a curvatura da coluna pela superfície externa, encontrou que a hiperlordose lombar se associou negativamente à flexibilidade lombar de forma geral. Ainda, Edmondston et al. (2011) em estudo realizado com 40 pessoas de ambos os sexos, com idade compreendida entre 18 a 30 anos, verificou correlação fraca, porém significativa entre o ângulo da cifose torácica e a amplitude de extensão torácica. A partir desses resultados, pode-se sugerir que, quando o indivíduo adquire uma posição em maior curva cifótica ou lordótica, ele reduz a sua capacidade de gerar movimento da coluna vertebral no mesmo sentido da curva e aumenta a capacidade de gerar movimento para o sentido oposto, provavelmente pelo fato de que a medição considera a posição inicial para o cálculo da ADM.

No entanto, cabe ressaltar que no presente estudo foram encontradas correlações entre a flexibilidade e a posição estática da coluna vertebral apenas quando utilizado como referência estática os IC e IL, mensurados na superfície da pele com o Flexicurva. Hinman (2004) utilizou o IC medido na posição relaxada e na posição ereta para avaliar a rigidez da coluna vertebral, e descreveu que, quanto maiores as taxas do IC, maior era rigidez na amostra estudada. Nesse sentido, parece que os índices são bons indicadores de flexibilidade da coluna vertebral até mesmo com medições apenas na posição relaxada, visto as correlações encontradas no presente estudo.

Há relatos de que a alteração da flexibilidade em outras regiões do corpo também pode influenciar a postura estática da coluna vertebral. Coelho et al. (2014) ao conduzir um estudo com escolares identificou que a flexibilidade de isquiotibiais apresentou influência sobre a inclinação anteroposterior corporal, sendo que os escolares com flexibilidade reduzida apresentaram maior inclinação corporal anteroposterior. Outros autores também relatam que a redução da flexibilidade desse grupo muscular pode ocasionar inclinação pélvica anteroposterior, afetando indiretamente a lordose lombar (CARREGARO; SILVA; COURY, 2007). Ainda, sabendo-se que a caracterização da flexibilidade de um indivíduo é multifatorial, entende-se necessário uma avaliação criteriosa para tornar possível a compreensão dos fatores que influenciam essa variável, de modo a possibilitar uma adequada intervenção, principalmente quando se trata de um sistema musculoesquelético complacente, como o da criança. Senthil et al. (2017) sugerem que o encurtamento do músculo peitoral menor poderia influenciar na posição da coluna torácica, gerando acentuação dessa curvatura. Hawke; Rome e Evans (2016) ao avaliar a relação entre a postura do pé e a flexibilidade em crianças, encontrou que crianças que apresentavam tipo de pé mais pronado exibiram maior flexibilidade do membro inferior, mas não da articulação no tornozelo.

Ao analisar separadamente os sexos, foi possível verificar diferença significativa quanto as ADMs, sendo encontrada maior ADM de flexão e total da região torácica no sexo feminino e maior ADM de flexão lombar no sexo masculino. Essa diferença pode ser explicada pelas diferenças culturais entre meninos e meninas, como por exemplo, a prática de diferentes atividades esportivas (COELHO et al., 2014). Acredita-se que a caracterização da flexibilidade de um indivíduo é multifatorial, sofrendo influência da idade, gênero, prática de exercício físico e variáveis antropométricas (ARAÚJO, 2008; GRANT et al., 2001).

Quanto à postura estática, não foram encontradas diferenças significativas entre os sexos, embora os meninos tenham sempre apresentado valores ligeiramente maiores. Resultados diferentes são descritos por Lemos, Santos e Gaya (2012), em que as meninas apresentam maior ocorrência de hiperlordose lombar $(84,5 \%)$ do que os meninos $(73,1 \%)$, havendo associação significativa entre o sexo e a hiperlordose lombar. Não obstante, entende-se que essa contradição entre os estudos carece de maior investigação para ser compreendido.

Dessa maneira, ressaltando a complexidade das variáveis analisadas, postura estática e flexibilidade, entende-se como limitação deste estudo a falta de controle sobre as demais variáveis que apresentam influência sobre a flexibilidade, tais como idade, gênero, prática de exercício físico e variáveis antropométricas. Além disso, o fato de não ter sido avaliada a flexibilidade de outras regiões corporais, como os isquiotibiais, por exemplo, pode ser considerada outra limitação do presente estudo.

Os achados desse estudo contribuem na prática clínica uma vez que demonstram aos profissionais da saúde a possibilidade de utilização de uma metodologia de avaliação da postura estática e da flexibilidade da coluna vertebral por um instrumento não invasivo, de baixo custo e que fornece resultados quantitativos, o que permite acompanhamento da evolução de um tratamento. Ainda, outra aplicação prática desse estudo refere-se ao ambiente escolar ou de academia, onde o profissional de Educação Física poderia facilmente aplicar essa metodologia para uma avaliação de base das crianças, a cada ano letivo, identificando possíveis alterações posturais ou de limitação da ADM da coluna vertebral, ou ainda, para potencializar a prescrição de um treinamento de flexibilidade, respectivamente. Considerando que índices maiores de cifose ou lordose e ADMs limitadas não são desejáveis, a utilização dessa metodologia de avaliação poderia servir como meio de triagem e controle nesses ambientes. Por fim, essa metodologia poderia também ser utilizada em estudos que investigassem o efeito de treinamentos de flexibilidade sobre a postura estática da coluna vertebral, o que por sua vez, contribuiria para uma melhor compreensão da relação entre essas variáveis.

\section{Considerações Finais}

Com base nos achados, não é possível afirmar que o ângulo de Cobb de cifose e lordose está relacionado com a flexibilidade da coluna vertebral. No entanto, a postura 
da coluna vertebral, dada pelos índices de cifose e lordose, se correlaciona com a flexibilidade da coluna vertebral, de modo que quanto maior a cifose torácica, menor é a ADM de flexão torácica e maior é a $\mathrm{ADM}$ de extensão torácica. De modo inverso, quanto maior é a lordose lombar, maior é a ADM de extensão lombar e menor é a ADM de flexão lombar. A comparação entre os sexos demonstrou que os meninos possuem maiores valores de ADM de flexão lombar enquanto as meninas possuem maiores valores de ADM de flexão torácica.

\section{Referências}

ARAÚJO, C. G. S. Flexibility assessment: normative values for flexitest from 5 to 91 years of age. Arq. Bras. Cardiol. v. 90, n. 2, p. 257-263, 2008.

BOSEKER, E. H. et al. Determination of "normal" thoracic kyphosis: A roentgenographic study of 121 "normal" children. J Pediatr Orthop. v. 20, n. 6, p. 796-798, 2000.

BURTON, K. A. Regional lumbar sagittal mobility: measurement by flexicurves. Clin Biomech. v. 1, n. 1, p. 20-26, 1986.

BYRD, S. E.; COMISKEY, E. M. Postnatal maturation and radiology of the growing spine. Neurosurg Clin N Am. v. 18, p. 431-461, 2007.

CARREGARO, R. L.; SILVA, L. C.; COURY, H. J. G. Comparison between two clinical tests for evaluating the flexibility of the posterior muscles of the thigh. Rev Bras Fisioter. v. 11, n. 2, p. 139-145, 2007.

CIL, A. et al. The evolution of sagittal segmental alignment of the spine during childhood. Spine, v. 30, n. 1, p. 93-100, 2005.

COELHO, J. J. et al. Influência da flexibilidade e sexo na postura de escolares. Rev Paul Pediatr. v. 32, n. 3, p. 223 $228,2014$.

EDMONDSTON, S. J. et al. Thoracic spine extension mobility in young adults: influence of subject position and spinal curvature. J Orthop Sports Phys Ther. v. 41, n. 4, p. 266-273, 2011.

FIELD, A. Descobrindo a estatística usando o SPSS. 2. ed. Porto Alegre: Artmed, 2009.

GONÇALVES, G. B.; PEREIRA, J. S. Radiological assessment of the angular values of back-lumbar and sacrallumbar curvature in adolescents. Acta fisiatr. v. 15, n. 1, p. 92-95, 2008.

GRANT, S. et al. A comparison of the anthropometric, strength, endurance and flexibility characteristics of female elite and recreational climbers and non-climbers. J Sports Sci. v. 19, n. 7, p. 499-505, 2001.

HAWKE, F.; ROME, K.; EVANS, A. M. The relationship between foot posture, body mass, age and ankle, lower-limb and whole-body flexibility in healthy children aged 7 to 15 years. J Foot Ankle Res. v. 9, n. 14, p. 1-5, 2016.

HINMAN, M. R. Interrater reliability of flexicurve postural measures among novice users. J Back Musculoskelet Rehabil. v. 17, n. 1, p. 33-36, 2004.

KADONO, N. et al. A japanese stretching intervention can modify lumbar lordosis curvature. Clin Spine Surg. v. 30, n. 7, p. 297-300, 2017.

KOTRLIK, J. W.; WILLIAMS, H. A. The incorporation of effect size in information technology, learning, and performance research. Information Technology, Learning and Performance J. v. 21, n. 1, p. 1-7, 2003.

LEMOS, A. T.; SANTOS, F. R.; GAYA, A. C. A. Hiperlordose lombar em crianças e adolescentes de uma escola privada no Sul do Brasil: ocorrência e fatores associados. Cad. Saúde Pública, v. 28, n. 4, p.781-788, 2012.

MALMSTRÖM, E. M. A slouched body posture decreases arm mobility and changes muscle recruitment in the neck and shoulder region. Eur J Appl Physiol. v. 115, n. 12, p. 2491-2503, 2015.

OLIVEIRA, T. S. et al. Validity and reproducibility of the measurements obtained using the flexicurve instrument to evaluate the angles of thoracic and lumbar curvatures of the spine in the sagittal plane. Rehabil Res Pract. v. 2012, p. $1-9,2012$.

PENHA, P. J.; JOÃO, S. M. A. Avaliação da flexibilidade muscular entre meninos e meninas de 7 e 8 anos. Fisioter Pesqui. v. 15, n. 4, p. 387-391, 2008.

PROPST-PROCTOR, S. L.; BLECK, E. E. Radiographic determination of lordosis and kyphosis in normal and scoliotic children. J pediatr orthop. v. 3, n. 3, p. 344-346, 1983.

SENTHIL, P. et al. Efficacy of corrective exercise strategy in subjects with hyperkyphosis. J Back Musculoskelet Rehabil, v. 30, n. 6, p. 1285-1289, 2017.

WIDHE, T. Spine: posture, mobility and pain. A longitudinal study from childhood to adolescence. Eur Spine J. v. 10, n. 2, p.118-123, 2001. 